\title{
Conceptualising barriers to incident reporting: a psychological framework
}

\author{
Y Pfeiffer, T Manser, T Wehner
}

Center for Organizational and Occupational Sciences, ETH Zurich, Zurich, Switzerland

\section{Correspondence to}

Dr Yvonne Pfeiffer, Center for Organizational and Occupational Sciences, ETH Zurich, Kreuzplatz 5, Zurich $\mathrm{CH}-8032$, Switzerland; ypfeiffer@ethz.ch

Accepted 26 July 2009 Published Online First 17 June 2010

\begin{abstract}
Background Incident reporting systems are widely considered effective instruments for learning from incidents. However, research shows that many incidents are not reported by healthcare providers.

Objective The lack of theoretical foundation in research on barriers to and motivators for incident reporting is addressed in this article, and a psychological framework of antecedents to staff's motivation (not) to report incidents is proposed.
\end{abstract}

Framework development Concepts relevant for clinicians' motivation to report incidents were identified in psychological literature. Additionally, a literature review was conducted to extract barriers to incident reporting and cluster them into thematic groups. Barriers and motivators influencing clinicians' willingness to report were integrated and identified as an indicator for actual reporting behaviour.

Conclusions The proposed framework provides a basis for guiding future empirical studies that will improve our understanding of what encourages and what hinders clinicians to report incidents and, consequently, of areas for interventions to enhance reporting behaviour.

In healthcare and other high-risk industries, incident reporting systems (IRS) are considered effective instruments to learn from adverse events, errors and near misses-hereafter referred to as incidents. ${ }^{1}$ Incidents provide "free lessons" on latent failures in organisations, ${ }^{2}$ allowing for potential threats to safety to be diagnosed before an accident occurs. IRS are increasingly implemented in healthcare and are often expected to positively influence safety culture by increasing the awareness of patient safety issues in the front lines of healthcare. ${ }^{3}$

Studies on the reporting behaviour of healthcare providers have shown that under-reporting is a major problem of IRS. ${ }^{4}$ Voluntary reporting systems are estimated to capture about $10 \%$ of occurring incidents. ${ }^{67}$ Numerous studies have discussed the various reasons for low reporting rates. However, these studies have been mainly descriptive and only few have been based on theories. The first studies that proposed theoretical frameworks of incident reporting in healthcare focused on how IRS should be implemented from a technical and organisational perspective. ${ }^{8}$ It has been highlighted that more research is needed to identify the motivational antecedents of a clinicians' decision to (not) report. ${ }^{81011}$ Karsh et al ${ }^{8}$ argue that the current lack of theoretical foundation has impeded the development of a conceptual model of factors influencing the use of IRS.

This article aims at conceptualising motivational aspects of incident reporting-seen as a deliberate and voluntary act of an individual to use an organisational learning instrument-into a framework that integrates relevant theoretical concepts from psychology and barriers and motivators for incident reporting that have been reported in the literature. This psychological framework provides a basis for future empirical studies and, thus, for effective measures to improve reporting rates.

\section{FRAMEWORK DEVELOPMENT}

This article follows the three steps of framework development: (1) identification of key concepts in psychological literature considered to be relevant to the motivation to report incidents in healthcare, (2) review of publications on barriers and motivators to incident reporting and (3) integration of these framework components into an overarching scheme. Figure 1 provides an overview of in the procedure used to develop the framework.

\section{Selection of psychological concepts relevant to incident reporting}

The aim of this step was to identify psychological concepts that are relevant to the discussion on barriers to and motivators for incident reporting in hospitals. As prior research was very much focused on what hinders clinicians from reporting, the notion of barriers is preponderant in literature. In our literature review, we chose a broader approach to motivational aspects, including barriers as reasons for not reporting and reasons for reporting in the sense of motivators. For example, Garbutt et $a l^{12}$ showed that a non-punitive system can increase the physicians' willingness to report. In conceptualising why clinicians do (not) report incidents from a psychological perspective, we took the following approach: first, in contrast to prior research that focused primarily on attitudes towards incident reporting, we decided to base our framework on a psychological theory describing how attitudes influence behaviour to map the relevant motivational cognitions adequately (Theory of reasoned action). Second, in many healthcare systems, reporting is not part of defined work routines or professional guidelines but reporting to IRS is entirely voluntary. This led us to include psychological theories explaining the development of proactive behaviour that is not explicitly required in job descriptions or professional guidelines (Role identity). Third, incident reporting often involves that the reporter experiences or observes some kind of error. Therefore, we integrated psychological concepts addressing individual's attitudes towards errors and concepts describing organisational characteristics that foster reporting (Error orientation). Finally, the perception of organisational characteristics 


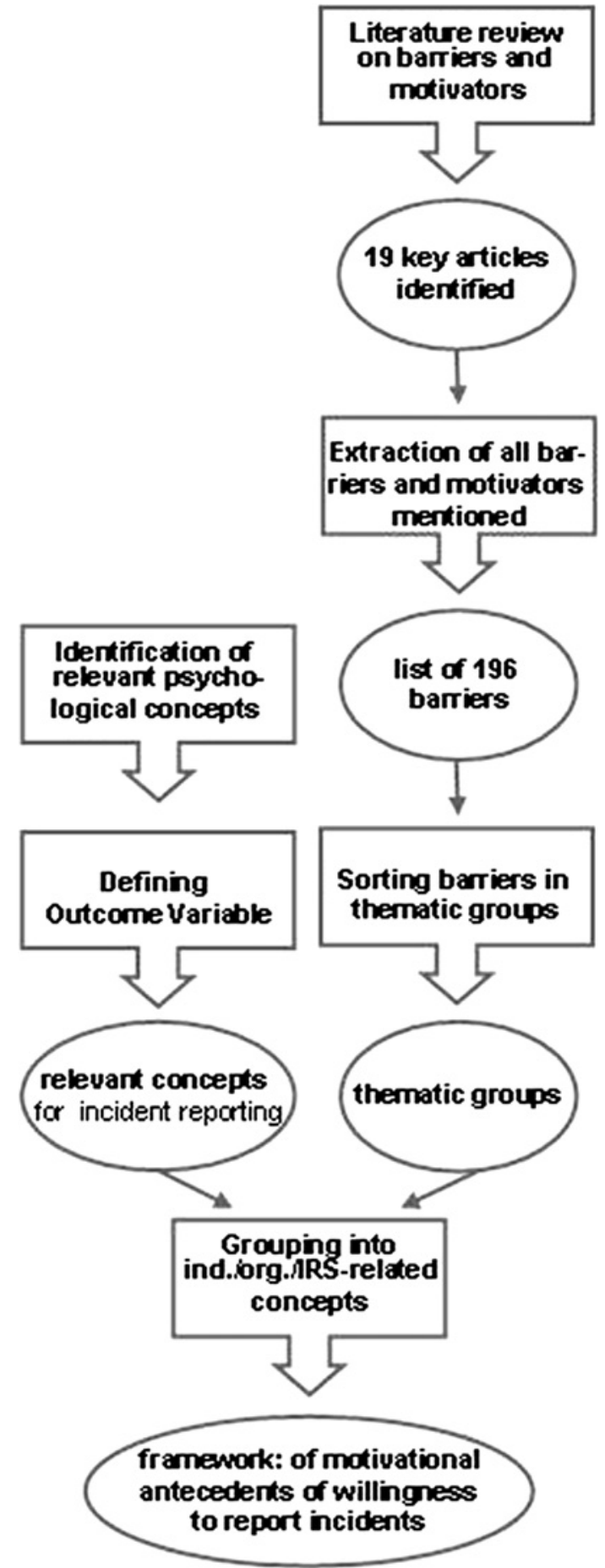

Figure 1 Research process of framework development.

plays a crucial role in clinicians' motivation to report incidents. ${ }^{8}$ To keep our framework as concise as possible, we focused on the three key concepts in the context of incident reporting: psychological safety, tolerance for organisational dissent and management support for patient safety.

Theory of reasoned action

The theory of reasoned action describes attitudes towards behaviours and how they relate to the intention to exert a behaviour. ${ }^{13}$ This well-established psychological theory is suited to grasp clinicians' beliefs regarding incident reporting and how they relate to the development of the intention to report incidents. The theory of reasoned action was designed to predict volitional behaviour or behaviours over which the individual has a great deal of control. ${ }^{14}$

The differentiation between attitudes and intention is useful because attitudes are not necessarily realised as behaviour and are discerned from the intention to report an incident, which is more proximal to behaviour. Thus, the theory of reasoned action is useful to bridge the attitude-behaviour gap, which is a problem in trying to predict actual reporting behaviour. Referring to the theory of reasoned action, we had the possibility to define a sound and measurable outcome variable: the intention to report, also referred to as "willingness to report", 15 in the following.

The theory of reasoned action also differentiates two influences on the intention to execute a specific behaviour: personal attitude and subjective norm. The personal attitude towards a specific behaviour consists of beliefs about possible consequences of the behaviour. The personal valuation of the consequences is weighted by their probability. Applying the theory of reasoned action to incident reporting, barriers stemming from the belief that the reporter's clinical competence is likely to be questioned after a report (ie, a consequence with a negative value) can be summarised under "personal attitudes". The intention to report an incident can be influenced by the perception that relevant others within the organisation (eg, colleagues, supervisors) expect this behaviour. This perception (ie, subjective norm) becomes relevant only if an individual is willing to comply with others' expectations. Subjective norm was shown to be important for the acceptance of an IRS in a study on barriers to incident reporting. ${ }^{9}$

\section{Role identity}

From a social identity theory perspective, Piliavin et $a l^{16}$ analysed different role identities to understand the (non-)reporting of incidents in healthcare. In general, role identity is developed when the role associated with a position in a social network has been internalised. In the context of professional behaviour, a "general role identity" (eg, "nurse") and "organisation-specific role identities" ("hospital employee", "member of a unit") are distinguished. ${ }^{17}$ With regard to reporting behaviour, these different role identities may conflict. For example, although a clinician's general role identity as physician or nurse might suggest reporting, he/she may not report because he/she feels committed to and fears negative consequences for his/her team (specific role identity as member of a unit).

\section{Error orientation}

As IRS aim at fostering learning from incidents including errors, reporting may be influenced by an individuals' attitude towards errors. Learning from errors is supported when staff has a positive attitude towards errors. ${ }^{18}$ Rybowiak et al ${ }^{18}$ developed a measure to assess error orientations, three of which seem especially relevant to incident reporting: (1) the assumption that one can learn from errors, (2) the tendency (not) to cover up errors and (3) the extent to which one perceives strain when errors occur.

\section{Psychological safety}

Psychological safety (ie, the extent to which staff is at ease to bring up own ideas and errors) has been shown to influence reporting behaviour ${ }^{19} 20$ and further learning from errors. ${ }^{21}$ It was therefore factored in our framework. Psychological safety is an essential element of a "just culture" 22 as it describes the expectation of a fair treatment when reporting an error. 
Tolerance for organisational dissent

Organisational dissent describes the phenomenon of bringing up tough issues or personal views that do not conform to established practices. ${ }^{23}$ These behaviours have been shown to be positively associated with safety performance. ${ }^{24} 25$ IRS offer the possibility to express personal concerns about safety issues and thus may serve as a formal dissent procedure as pointed out by Piliavin et al. ${ }^{16}$ Thus, we included the concept of "tolerance for organisational dissent"26 in our framework.

\section{Management support for patient safety}

Leadership has been shown to be a crucial success factor for incident reporting in numerous studies. ${ }^{9}{ }^{27-32}$ Thus, clinicians perceiving their management as being active in enhancing patient safety and fostering incident reporting are expected to be more willing to report.

\section{Literature review on barriers to incident reporting}

To identify papers on reasons for (not) reporting incidents, a literature search consulting the databases MEDLINE and PsychINFO was carried out. The following search terms were used: "adverse event, error, incident, sentinel event" combined with "report*" or "system". The publication period was not limited (termination of search: August 2008). From the identified articles matching our interest, we searched in the bibliographies to find more papers. Inclusion criteria were that the papers had to focus on attitudes towards incident reporting in healthcare, regardless of sample size, cultural location of the study and whether the study empirically or theoretically investigated barriers and motivators to incident reporting. Papers not dealing with hospital settings (such as Elder et al's ${ }^{3}$ ) and papers only alluding to but not focusing on barriers were excluded from further analysis. Publications were screened by two researchers who read all abstracts. Table 1 describes the 19 papers that matched the inclusion criteria.

\section{Extraction of barriers and motivators}

The review of the 19 articles that met the inclusion criteria resulted in a list of 196 mentions of barriers. For barrier extraction, the following rules were applied:

1. It was of no importance whether a certain barrier or motivator proved to be relevant in a study; it just had to be mentioned. This extraction rule was applied to get a comprehensive overview of the current scientific discussion on barriers and motivators to reporting because our framework ought to serve as a foundation for investigating barriers empirically.

2. For each article, a certain reason for (not) reporting was only extracted once, even when it was referred to several times within the same publication-for example, in results and discussion.

3. Barrier extraction was performed by two researchers to assure the objectiveness of the process. Differences were resolved by discussion between the researchers until consensus was reached how to best apply the extraction rules.

\section{Classification into thematic groups}

In sorting the extracted barriers and motivators into thematic groups, we adopted a consensus-oriented approach. One researcher sorted all extracted barriers in a bottom-up process into thematic groups. Then, a second researcher classified all barriers using the thematic groups already defined and added new thematic groups when necessary. Finally, the results of the two sorting processes were compared. Differences were discussed between the two raters and a third researcher specialised in patient safety until a consensus between all three researchers was reached. All researchers involved in this process were work and organisational psychologists. Inter-rater reliability was not calculated because the process was designed to be iterative and consensus oriented.

\section{Thematic groups of barriers and motivators to incident reporting}

Table 2 summarises the thematic groups of barriers and motivators extracted from these papers and sorted along the concepts of the framework. For example, the barriers "employees being unsure about the definition of what should be reported" or "what to report needs to be clearly defined" were assigned to one thematic group called "no clear definition of incident", which is part of the IRS-related perceptions in the framework. In fact, most reasons for (not) reporting mentioned in literature refer to IRS characteristics and their perception by potential users (46\%) or to personal attitudes (42\%). In our research, barriers and motivators stemming from the belief about the consequences of reporting were assigned to the category "personal attitude". For example, the belief that reporting may have legal consequences for the reporter was conceptualised as personal attitude and assigned to the thematic group "fear of lawsuits" (table 2). Three papers $^{13} 3447$ brought forth barriers that were subsumed to the thematic group "clinicians are not encouraged to make reports" and thus represent an aspect of the organisational dimension psychological safety. ${ }^{19}$

If one article referred to barriers covering various aspects of the same thematic group, each barrier mentioned was counted and sorted in this thematic group (see table 2, "times mentioned"). For example, Schectman and Plews-Ogan ${ }^{39}$ mentioned two barriers, "reporting too difficult" and "unsure of reporting mechanism", which we assigned to one group: "not knowing how to report an incident".

Furthermore, several studies mentioned the influence of incident characteristics on reporting behaviour.

\section{Integration into a framework}

In figure 2, the framework and how its components relate to each other are represented. The psychological concepts assumed to be relevant for incident reporting were combined with the results of the review of papers focusing on reasons for (not) reporting (see table 2). In the following, it is described how we proceeded in setting up the framework.

\section{Outcome variable willingness to report}

We defined "willingness to report" 15 as an outcome variable because the motivational antecedents of clinicians' reporting behaviour are the focus of our framework (see figure 2). In line with the theory of reasoned action, ${ }^{48}$ the willingness to report is regarded as an indicator for the intention to report incidents. This intention has been shown to be the best subjective predictor for actual reporting behaviour. ${ }^{15}$ In our framework, we propose to conceptualise barriers and motivators as influences on the willingness to report, in which "motivators" are defined as factors that enhance the willingness to report, whereas "barriers" decrease the willingness to report.

\section{Theoretical concepts relevant to the willingness to report}

To structure the concepts influencing the outcome, we broadly assigned them to groups: individual influences, organisational influences and influences related to the perception of the IRS. Separated from these perception-based influences, we also mapped the influences of the "objective" characteristics of incidents. The three concepts psychological safety, tolerance for 

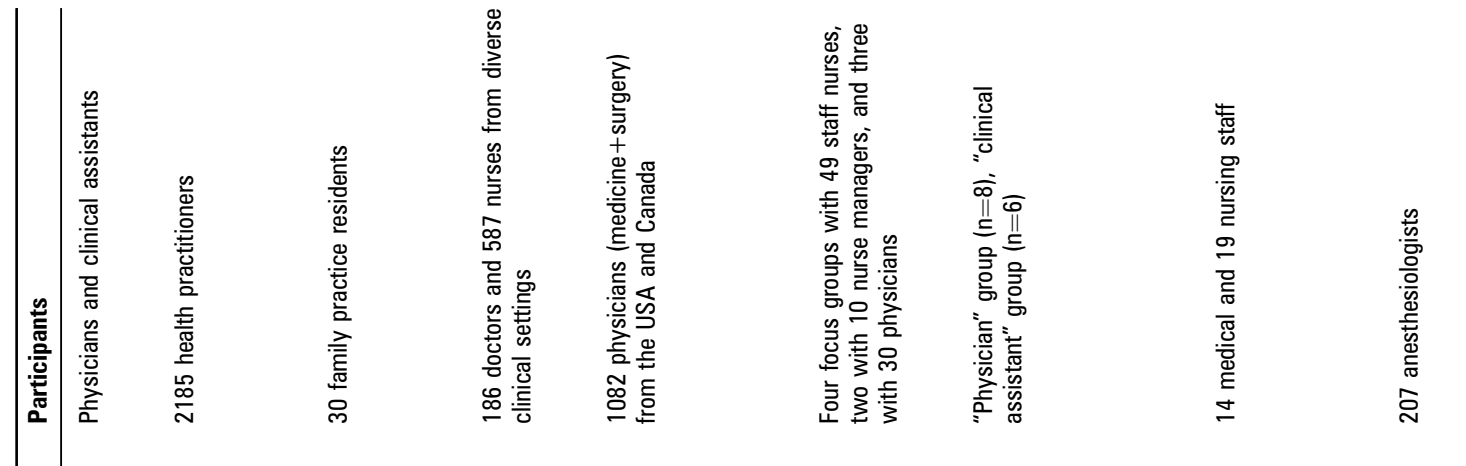

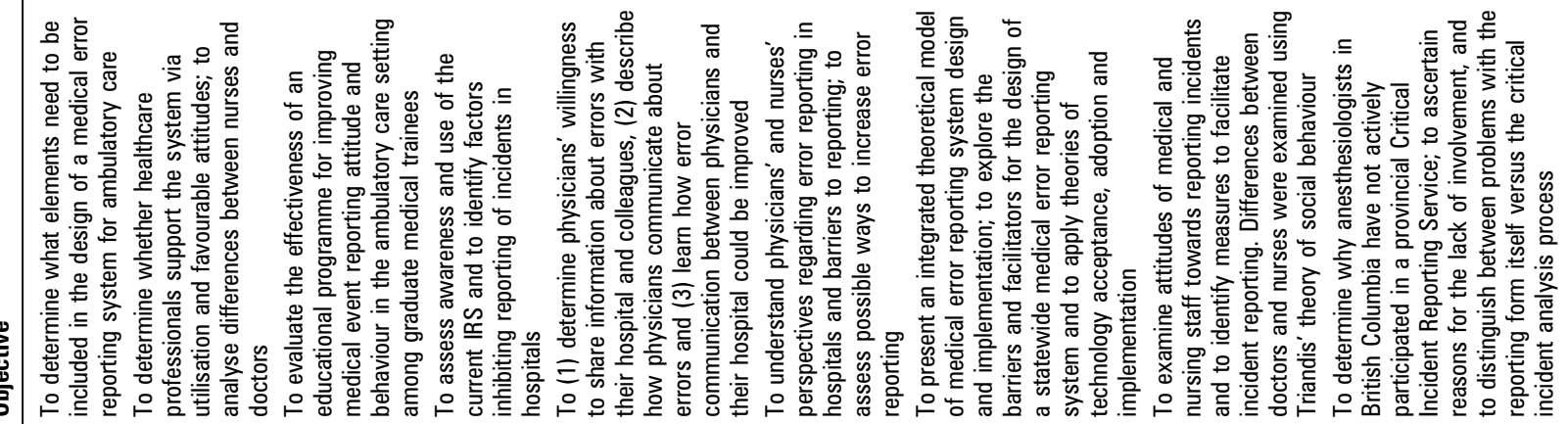

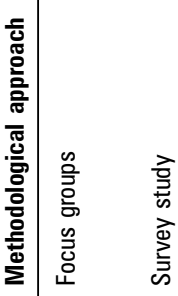
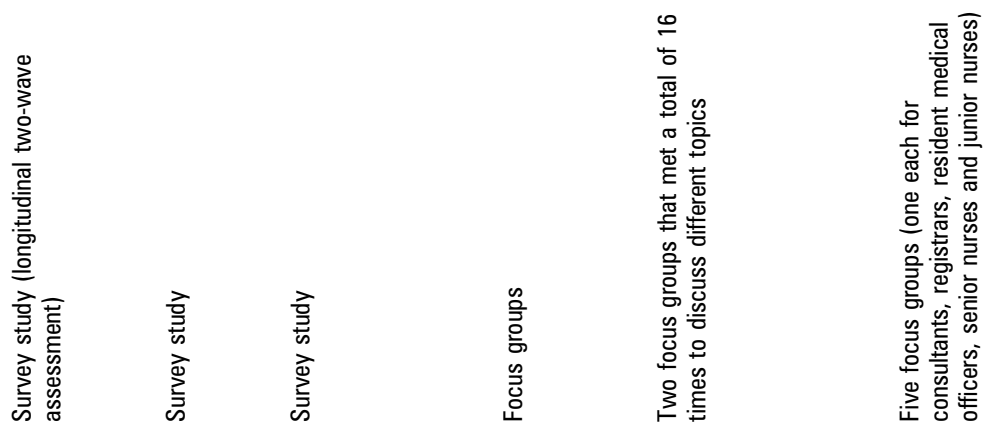

旁

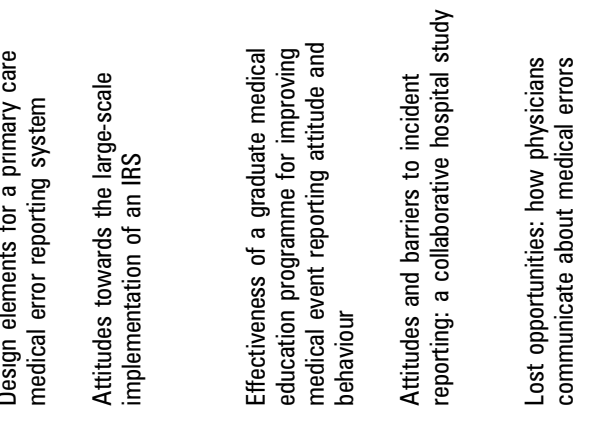
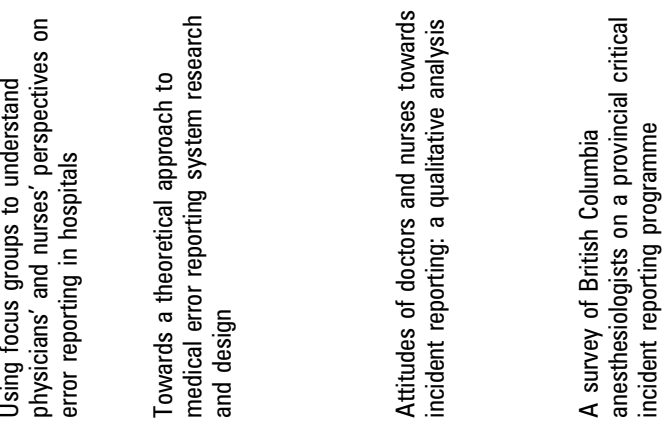

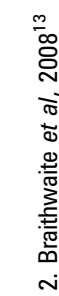

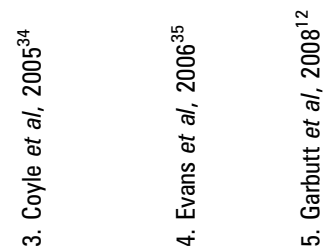

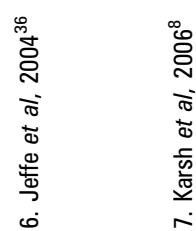

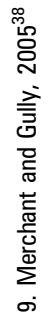




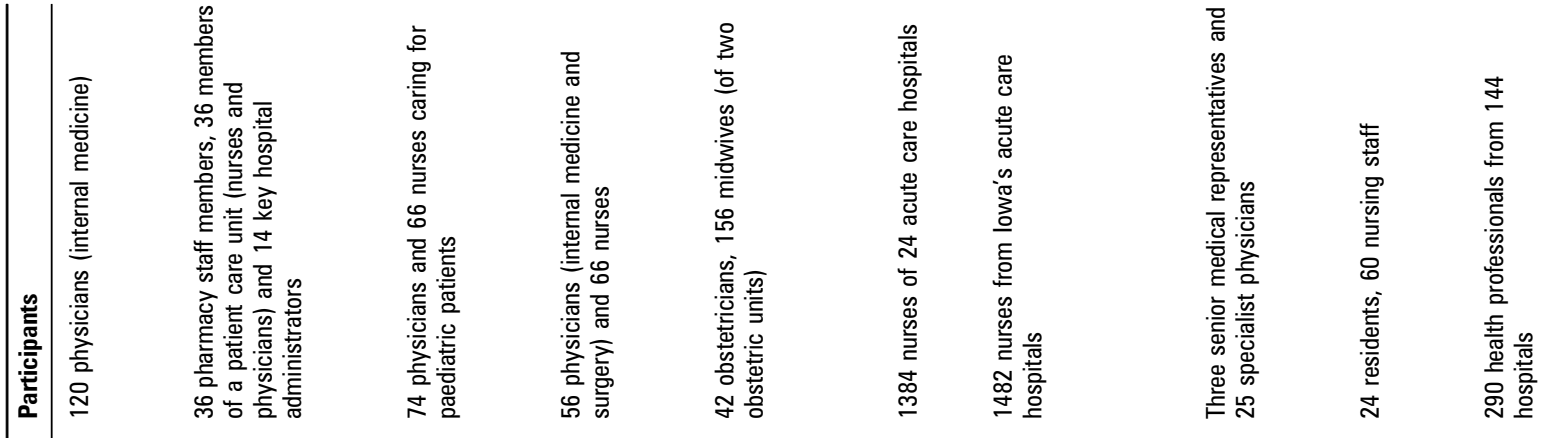

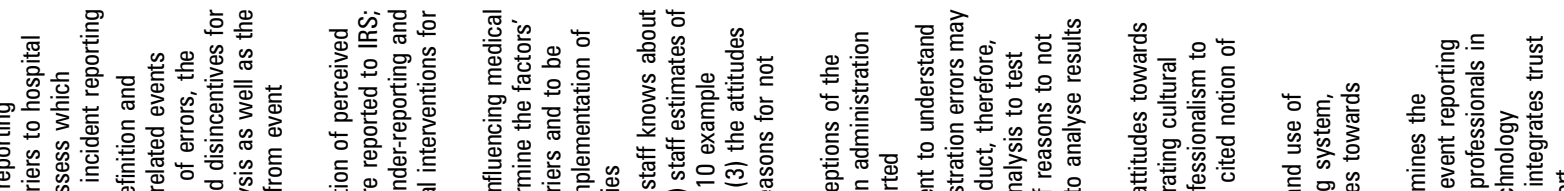
$\begin{array}{lllll} & \\ \end{array}$ $\begin{array}{llll} & \\ 5\end{array}$

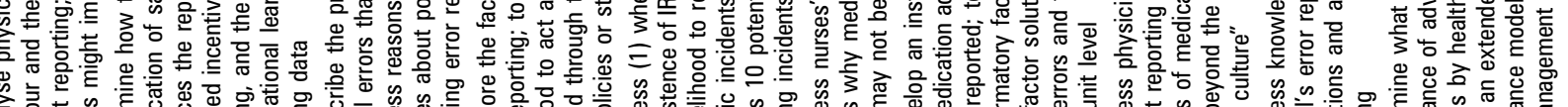

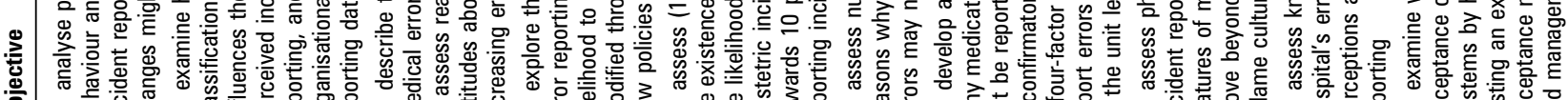

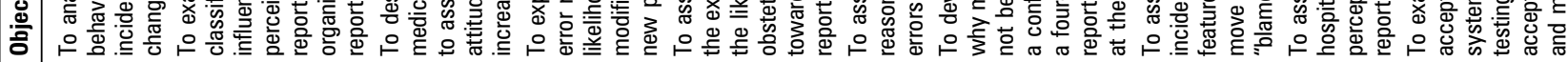

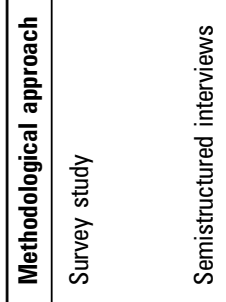

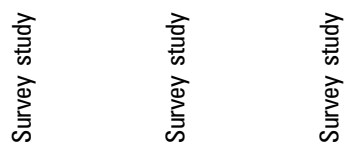
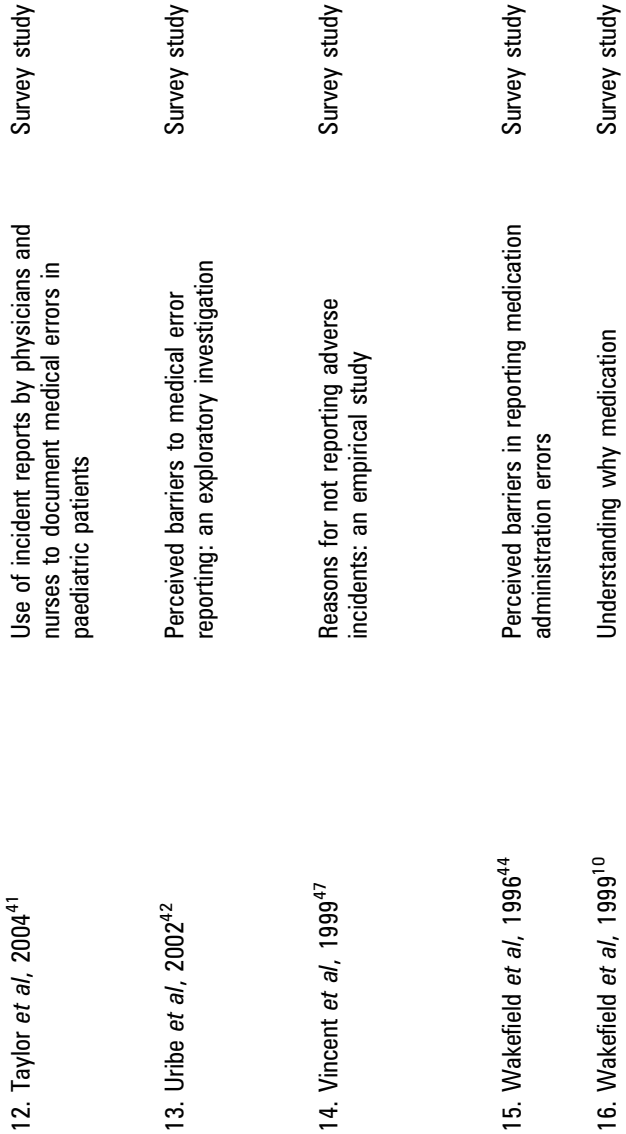

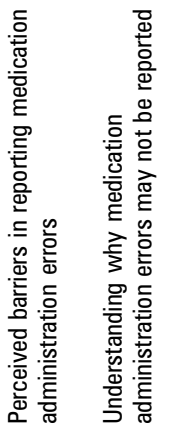

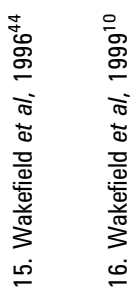

.
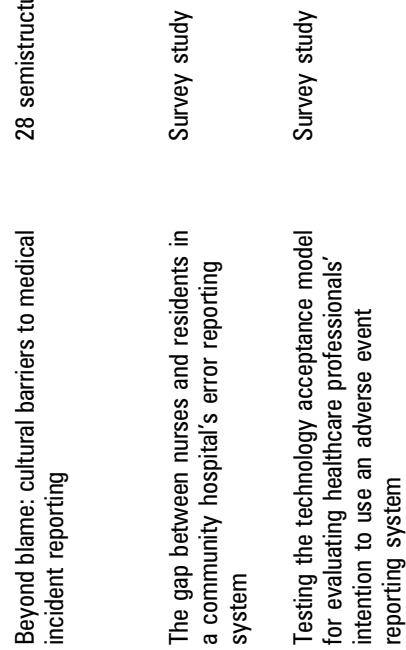

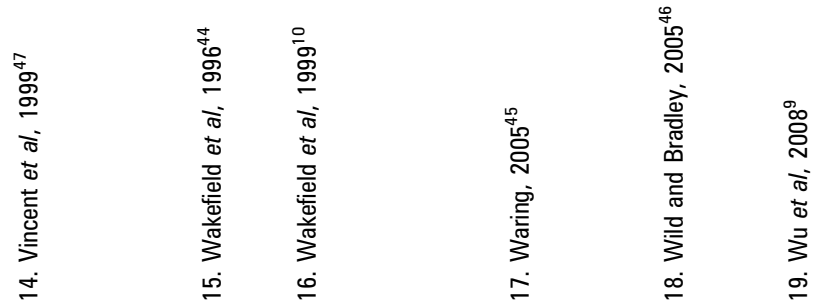




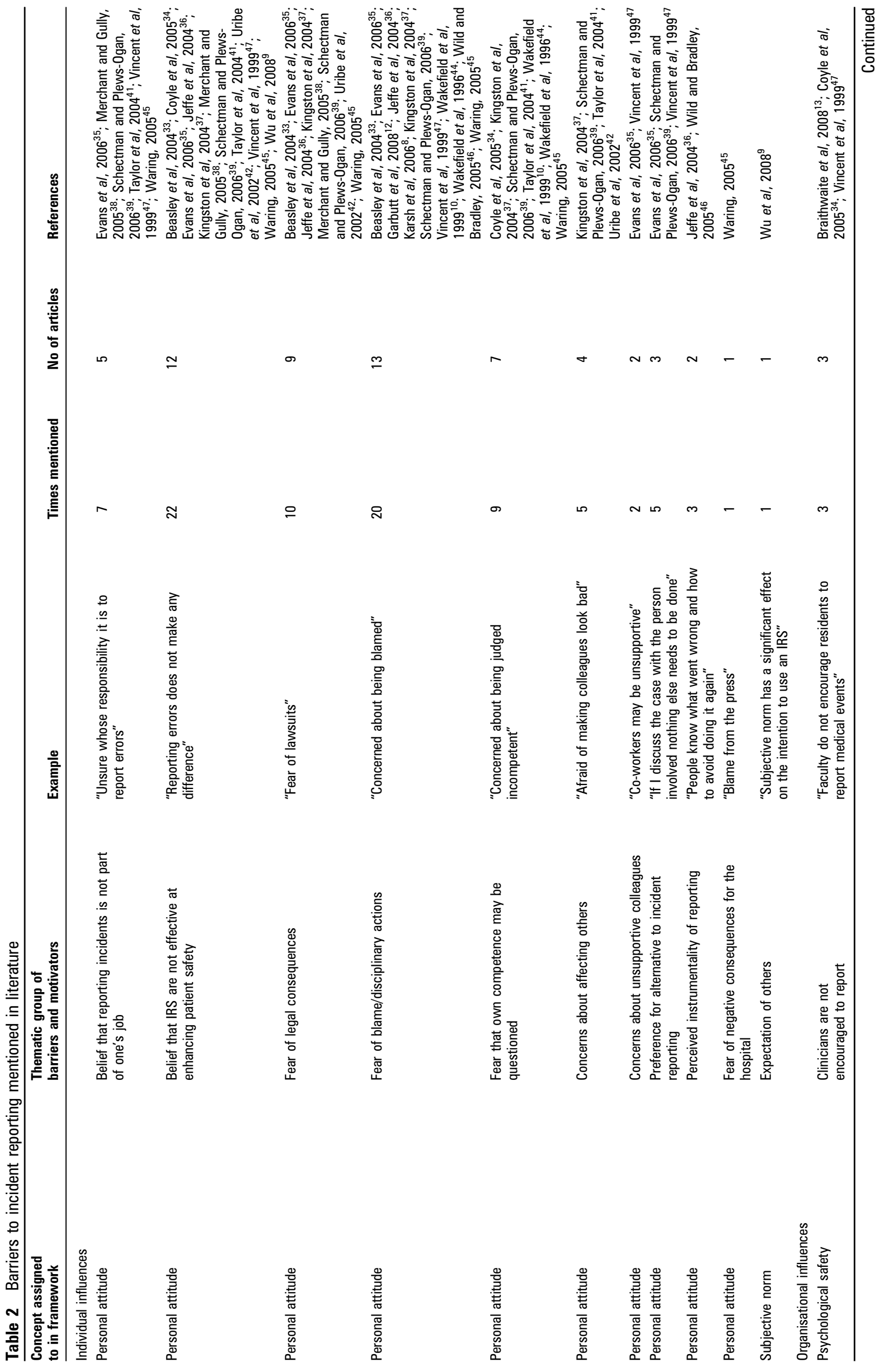

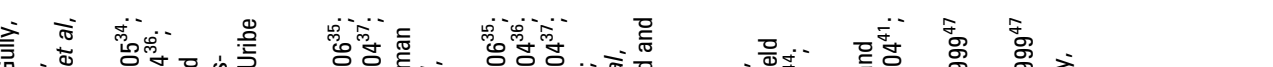

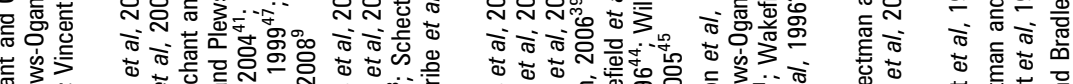

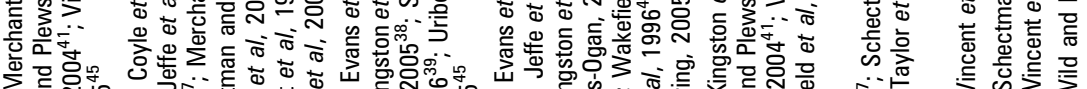

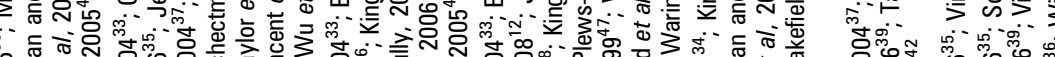

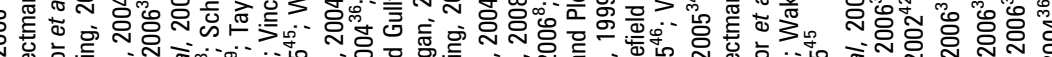

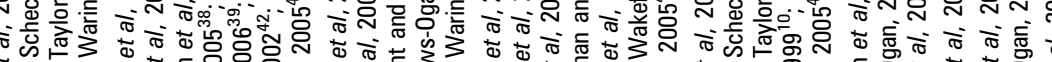

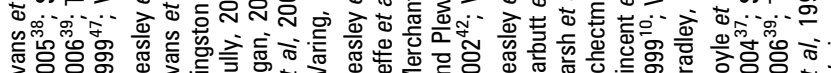

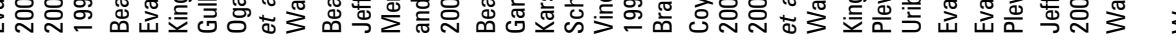




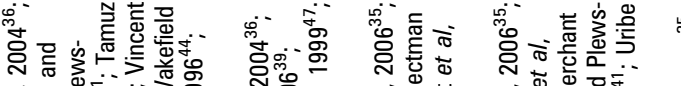

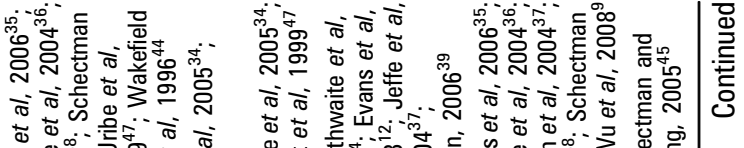

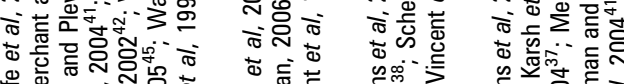

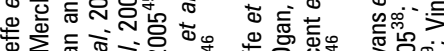

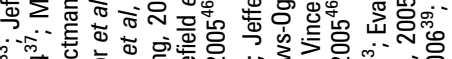

空

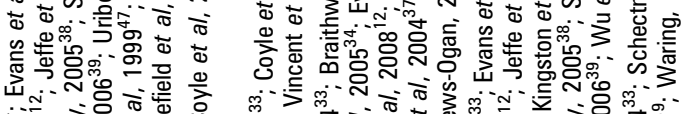

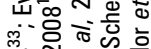

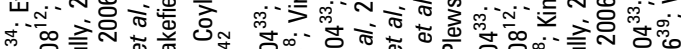

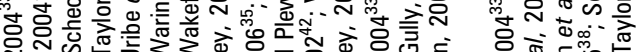

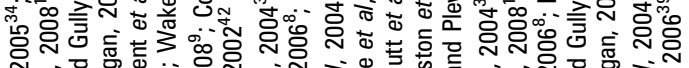

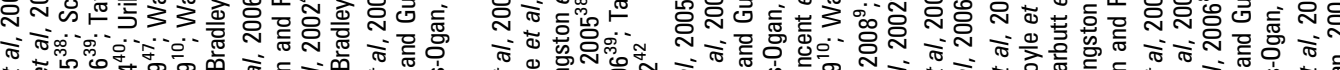

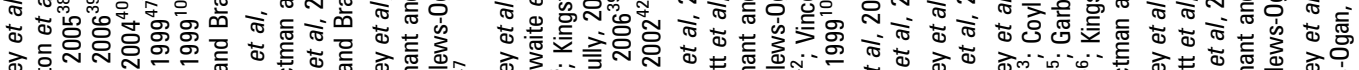

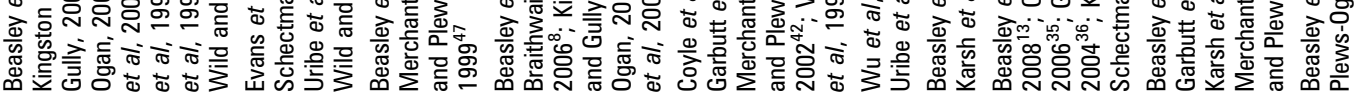

$=$

.

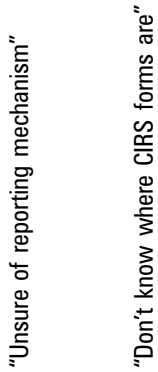

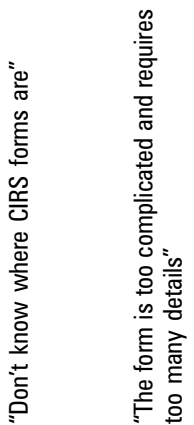

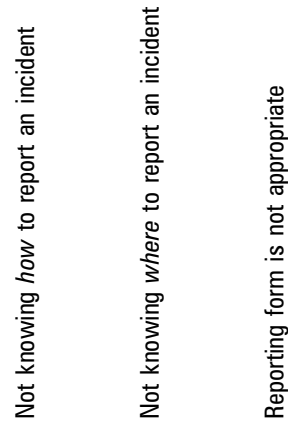

$\frac{\sqrt{\mathrm{d}}}{\mathrm{d}}$

高

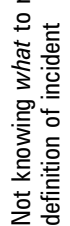

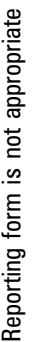

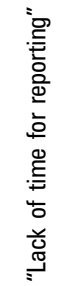

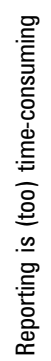

으

$\simeq$

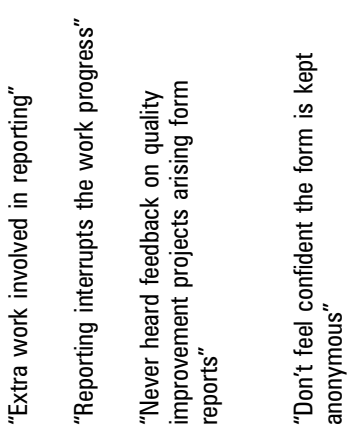

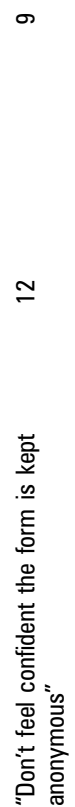
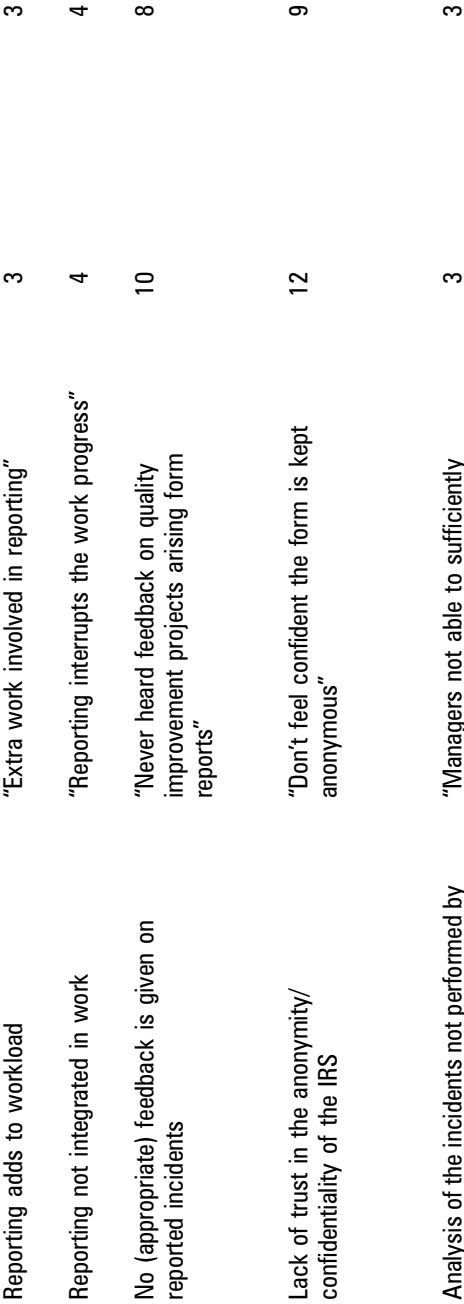

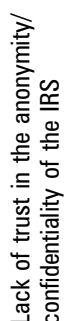

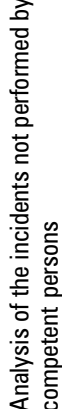




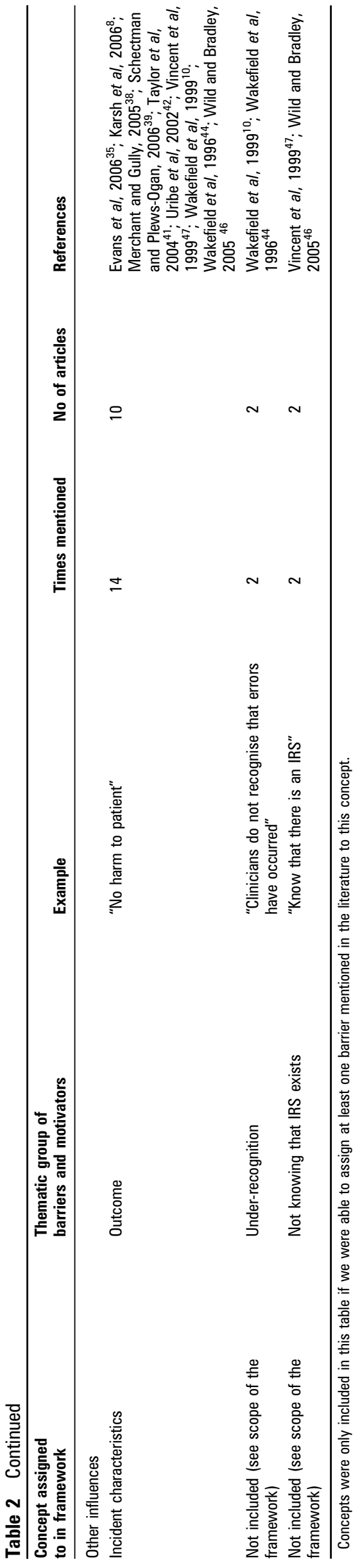

organisational dissent and management support for patient safety were grouped to the organisational influences assumed to be relevant for the willingness to report. The third group of relevant influences on the willingness to report are the "perception of IRS characteristics" (see figure 2).

\section{Incident characteristics}

In line with the literature, 8103538394142444647 we assume an influence of characteristics of incident such as severity, error type or involvement of the reporter on the reporting of an incident. This influence has been investigated by various studies-for example, by Lawton and Parker. ${ }^{49}$ They find that the outcome of an incident and whether a violation of a protocol was involved have an influence on the willingness to report. The study of Piliavin $e$ e $a l^{16}$ points to the fact that the motivation to report may vary when the incident was only observed from when the reporter was involved.

\section{Relation of concepts to each other}

Most barriers and motivators extracted from our review were assigned to the groups "perception of IRS characteristics" and "personal attitudes" sensu Fishbein and Ajzen. ${ }^{48}$

All concepts factored in the framework are assumed to influence the willingness to report incidents to an IRS. Additionally, personal attitudes have an important function because all dimensions - individual, organisational and IRS related-may influence the personal attitudes. Thus, the influence of all dimensions of the framework on the willingness to report may be mediated by personal attitudes. For example, we expect that personal attitudes become actualised according to the dominant type of role identity of an individual. Thus, the fear of negative consequences for the hospital (which is a personal attitude) can be actualised by a strong identification with the hospital (specific role identity as employee of a hospital). Furthermore, we expect characteristics of an incident to influence (1) clinicians' personal attitude towards reporting this incident and (2) the relationship between individual and organisational antecedents and the willingness to report (see figure 2).

\section{Scope of the framework}

In developing our framework, two barriers mentioned in literature were excluded (see table 2). First, staff may not know that an IRS exists in their organisation. ${ }^{46}{ }^{47}$ This barrier was excluded because it constitutes a communication problem and not a motivational antecedent for under-reporting. Second, clinicians may not report incidents because they did not recognise them. Under-recognition of incidents is a problem in healthcare 3104450 but the challenge is educational rather than motivational and is therefore not addressed by our framework. Consequently, our framework applies to clinical staff aware of the IRS deciding whether to report after having recognised an incident. Additionally, all organisational dimensions implied in the framework refer to perceptions on the work unit level as this is a management level highly relevant for the work in hospital settings. ${ }^{51}$ Finally, the framework applies to organisations in which incident reporting is voluntary.

\section{DISCUSSION}

The framework proposed in this article contributes to the development of a theoretical model and thus to an advanced understanding of factors influencing clinicians' reporting behaviour. Focusing on the motivational antecedents of incident reporting, it expands existing frameworks for the successful 
Figure 2 Psychological framework on factors influencing the willingness to report incidents. ${ }^{*}$, The full list of thematic groups is given in table 2 .

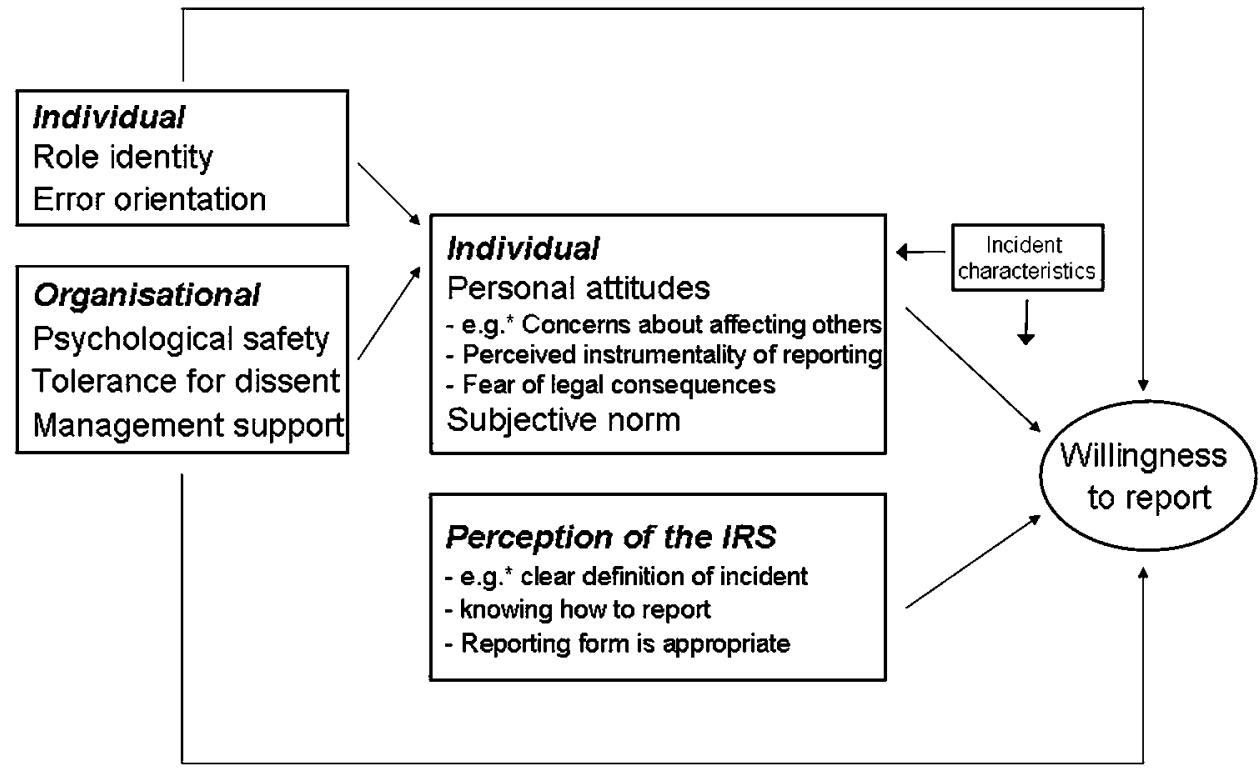

implementation of IRS by integrating behavioural theories. ${ }^{8} 11$ It contributes to the discussion of motivational influences on the willingness to report-including motivators enhancing the willingness to report as well as barriers inhibiting incident reporting.

The proposed framework provides a theoretically sound basis for further empirical studies of factors influencing clinicians' reporting behaviour. A guiding principle in framework development was to integrate concepts that are testable in a self-report survey for empirical investigation. Therefore, the concepts were factored as individual perceptions, out of the objective influence the type of incident may have. Empirical studies will provide the opportunity to validate the proposed framework by further testing the relevance of the psychological concepts included. This empirical investigation will lead to a refinement of the proposed framework. For example, the empirical studies may show that concepts have only mediated but no direct influence on the outcome or that some concepts are of such importance that they need to be addressed by the framework in a more finegrained way.

\section{Examination of professional differences}

For example, there is still a lack of theory-based evidence on professional differences in IRS use. ${ }^{13} 2737$ In an empirical study based on the proposed framework, multigroup analyses (in a structural equation model) will show whether there are motivational differences between groups of clinicians and whether different factors will enhance or reduce the willingness to report for physicians and nurses, respectively. This kind of study will also help to determine whether the extent to which a person feels associated to a general role identity as nurse or physician influences the motivation to report incidents. To this end, a hypothesis can be defined based on the theories integrated in our framework. For example, following Piliavin et $a l,{ }^{16}$ it can be assumed that nurses have different role identities than physicians and therefore other personal attitudes become relevant for their intention to report. Similarly, error orientations may differ between professional groups and may also actualise different personal attitudes. Differences in perceptions of IRS between physicians and nurses have been investigated before (see table 1). Similarly, this kind of analysis will be possible for the perception of organisational dimensions. These analyses will give insight in which conditions have to be changed for nurses and for physicians so that they really use IRS.

\section{IRS characteristics}

Furthermore, clinicians' perceptions of an IRS as well as their willingness to report may vary considerably depending on how the IRS is implemented and run within their organisation. IRS characteristics assumed to be relevant are degree of anonymity or confidentiality, ${ }^{52}{ }^{53}$ the reporting form (eg, paper based vs electronic), ${ }^{33} 3537$ the process for analysing incidents (eg, within hospital vs external experts), ${ }^{43}$ the definition and classification of incidents to be reported, ${ }^{40}$ and the feedback process on incidents reported and measures taken to prevent reoccurrence. ${ }^{34} 39$ According to the investigation of differences between professional groups, a cross-sectional study comparing hospitals using IRS that vary on the above-described dimensions will shed light onto the influences that the IRS itself has on the concepts of the framework and their interrelations.

\section{Incident characteristics}

Various aspects of the influence of incident characteristics on the reporting of incidents have been analysed. 8101635383941424446474950 Empirical investigation of these possible influences may be conducted by using vignettes describing an incident to examine their influence on the perceptions factored in the framework. Additional to a direct influence of incident characteristics on the willingness to report, we expect a moderating influence of incident

\section{Key messages}

- Considering barriers as influences on the willingness to report accounts also for positive motivators fostering incident reporting.

- Influences on reporting behaviour can be assigned to three groups: individual, organisational and IRS related.

- Integrating theories from psychology will advance the understanding of how staff is motivated to report incidents.

- The framework serves as a guiding basis for future empirical investigations. 
characteristics on the relationship between personal attitudes and the willingness to report (see figure 2). Evidence on how different types of incidents influence the motivation to report them provides useful information for further development and design of IRS.

In summary, these empirical studies will improve our understanding of what encourages and what hinders clinicians to report incidents. This will contribute to a more comprehensive approach for supporting healthcare organisations in successfully implementing IRS and in defining a strategy to improve incident reporting in an international context. ${ }^{13}$

Acknowledgements We thank Simon Foster for his support in the literature review. We also thank the reviewers who helped us significantly in the presentation of the framework.

Funding This study was funded by the Commission for Technology and Innovation (KTI 8614.1 ESPP-ES) and by an internal research grant of ETH Zurich.

Competing interests None.

Provenance and peer review Not commissioned; externally peer reviewed.

\section{REFERENCES}

1. Runciman WB, Sellen A, Webb RK, et al. Errors, incidents, and accidents in anaesthetic practice. Anaesth Intensive Care 1993:21:506-19.

2. Reason J. Human error. New York: Cambridge University Press, 1990

3. Elder NC, Graham D, Brandt E, et al. Barriers and motivators for making error reports from family medicine offices: a report from the American Academy of Family Physicians National Research Network (AAFP NRN). J Am Board Fam Med 2007; 20:115-23.

4. Stanhope N, Crowley-Murphy M, Vincent C, et al. An evaluation of adverse incident reporting. J Eval Clin Pract 1999;5:5-12.

5. Schuerer DJE, Nast PA, Harris CB, et al. A new safety event reporting system improves physician reporting in the surgical intensive care unit. J Am Coll Surg 2006;202:881-7.

6. Jones KJ, Cochran GL, Hicks RW, et al. Translating research into practice: voluntary reporting of medication errors in critical access hospitals. J Rural Health 2004;20: 335-43.

7. Barach $\mathbf{P}$, Small S. Reporting and preventing medical mishaps: lessons from nonmedical near miss reporting systems. Br Med J 2000;320:759-63.

8. Karsh B-T, Escoto KH, Beasley JW, et al. Toward a theoretical approach to medical error reporting system research and design. App/ Ergon 2006:37:283-95.

9. Wu JH, Shen WS, Lin LM, et al. Testing the technology acceptance model for evaluating healthcare professionals' intention to use an adverse event reporting system. Int J Qual Health Care 2008;20:123-9.

10. Wakefield DS, Wakefield BJ, Uden-Holman T, et al. Understanding why medication administration errors may not be reported. Am J Med Qual 1999:14:81-8.

11. Holden RJ, Karsh BT. A review of medical error reporting system design considerations and a proposed cross-level systems research framework. Hum Factors 2007:49:257-76.

12. Garbutt J, Waterman AD, Kapp JM, et al. Lost opportunities: how physicians communicate about medical errors. Health Aff 2008;27:246-55.

13. Braithwaite $\mathbf{J}$, Westbrook M, Travaglia J. Attitudes toward the large scale implementation of an incident reporting system. Int J Qual Health Care 2008;20: 184-91.

14. Langdridge D, Sheeran P, Connolly KJ. Analyzing additional variables in the theory of reasoned action. J Appl Soc Psychol 2007;37:1884-913.

15. Edmondson AC. Learning from mistakes is easier said than done: group and organizational influences on the detection and correction of human error. $J \mathrm{App}$ Behav Sci 1996;32:5-28

16. Piliavin JA, Grube JA, Callero PL. Role as resource for action in public service. J Soc Issues 2002; $\mathbf{5 8 : 4 6 9 - 8 5 . ~}$

17. Grube JA, Piliavin JA. Role identity, organizational experiences, and volunteer performance. Pers Soc Psychol Bull 2000;26:1108-19.

18. Rybowiak V, Garst H, Frese M, et al. Error orientation questionnaire (EOO): reliability, validity, and different language equivalence. J Organ Behav 1999:20:527-47.

19. Edmondson A. Psychological safety and learning behavior in work teams. Admin Sci Quart 1999;44:350-83.

20. Edmondson AC. Learning from failure in health care: frequent opportunities, pervasive barriers. Qual Saf Health Care 2004;13 (Suppl 2):ii3-9.
21. Carmeli A. Social capital, psychological safety and learning behaviours from failure in organisations. Long Range Plann 2007;40:30-44.

22. Weiner BJ, Hobgood C, Lewis MA. The meaning of justice in safety incident reporting. Soc Sci Med 2008:66:403-13.

23. Shahinpoor N, Matt B. The power of one: dissent and organizational life. J Bus Ethics 2007:74:37-48.

24. Neal A, Griffin MA, Hart PM. The impact of organizational climate on safety climate and individual behavior. Saf Sci 2000;34:99-109

25. Weick KE. Organizational culture as a source of high reliability. Calif Manage Rev 1987;29:112

26. Kassing JW. From the looks of things: assessing perceptions of organizational dissenters. Manage Comm Quart 2001:14:442-70.

27. Johnson C. Human factors of health care reporting systems. In: Carayon P, ed. Handbook of human factors and ergonomics in healthcare and patient safety. New Jersey: Mahwah, 2007:525-60.

28. Pronovost PJ, Thompson DA, Holzmueller CGL, et al. Toward learning from patien safety reporting systems. J Crit Care 2006;21:305-15.

29. Flin R, Yule S. Leadership for safety: industrial experience. Qual Saf Health Care 2004; 13:ii45-51

30. Firth-Cozens J, Mowbray D. Leadership and the quality of care. Qual Saf Health Care 2001;10:ii3-7.

31. Guldenmund FW. The nature of safety culture: a review of theory and research. Saf Sci 2000:34:215-57.

32. Guldenmund FW. The use of questionnaires in safety culture research-an evaluation. Saf Sci 2007:45:723-43.

33. Beasley JW, Escoto KH, Karsh BT. Design elements for a primary care medical error reporting system. WMJ 2004;103:56-9.

34. Coyle YM, Mercer SO, Murphy-Cullen CL, et al. Effectiveness of a graduate medica education program for improving medical event reporting attitude and behavior. Qual Saf Health Care 2005; 14:383-8.

35. Evans SM, Berry JG, Esterman AJ, et al. Attitudes and barriers to incident reporting: a collaborative hospital study. Oual Saf Health Care 2006;15:39-43.

36. Jeffe DB, Dunagan WC, Garbutt J, et al. Using focus groups to understand physicians' and nurses' perspectives on error reporting in hospitals. Jt Comm J Oual Saf 2004;30:471-9

37. Kingston MJ, Evans SM, Smith BJ, et al. Attitudes of doctors and nurses towards incident reporting: a qualitative analysis. Med J Aust 2004;181:36-9.

38. Merchant RN, Gully PM. A survey of British Columbia anesthesiologists on a provincial critical incident reporting program. Can J Anesth 2005:52:680-4.

39. Schectman JM, Plews-Ogan ML. Physician perception of hospital safety and barriers to incident reporting. Jt Comm J Qual Patient Saf 2006;32:337-43.

40. Tamuz M, Thomas EJ, Franchois KE. Defining and classifying medical error: lessons for patient safety reporting systems. Qual Saf Health Care 2004;13:13-20.

41. Taylor JA, Brownstein D, Christakis DA, et al. Use of incident reports by physicians and nurses to document medical errors in pediatric patients. Pediatrics 2004:114: 729-35

42. Uribe CL, Schweikhart SB, Pathak DS, et al. Perceived barriers to medical-error reporting: an exploratory investigation. J Healthc Manag 2002;47:263-79.

43. Vincent C. Understanding and responding to adverse events. $N$ Eng/ J Med 2003;348:1051-6.

44. Wakefield DS, Wakefield BJ, Uden-Holman T, et al. Perceived barriers in reporting medication administration errors. Best Pract Benchmarking Healthc 1996;1:191-7.

45. Waring JJ. Beyond blame: cultural barriers to medical incident reporting. Soc Sci Med 2005;60:1927-35

46. Wild D, Bradley EH. The gap between nurses and residents in a community hospital's error-reporting system. Jt Comm J Qual Patient Saf 2005;31:13-20.

47. Vincent C, Stanhope N, Crowley-Murphy M. Reasons for not reporting adverse incidents: an empirical study. J Eval Clin Pract 1998;5:13-21.

48. Fishbein M, Ajzen I. Belief, attitude, intention, and behavior: an introduction to theory and research. Reading (MA): Addison-Wesley, 1975.

49. Lawton R, Parker D. Barriers to incident reporting in a healthcare system. Qual Saf Health Care 2002:11:15-8.

50. Jayasuriya JP, Anandaciva S. Compliance with an incident report scheme in anaesthesia. Anaesthesia 1995:50:846-9.

51. Gaba DM, Singer SJ, Rosen AK. Safety culture: is "unit" the right "unit of analysis"? Crit Care Med 2007:25:314-6.

52. Andersen HB, Madsen MD, Hermann N, et al. Reporting adverse events in hospitals: a survey of the views of doctors and nurses on reporting practices and models of reporting. In: Johnson C, ed. Investigation and reporting of incidents and accidents. Glasgow (UK): GIST Technical Report, G2002-2, 2002. 127-36.

53. Kaplan H, Barach P. Incident reporting: science or protoscience? Ten years later. Qual Saf Health Care 2002;11:144-5. 